\title{
Simulation of the scattering properties of a chain-forming triangular prism oceanic
}

diatom

Bingqiang Sun, ${ }^{1}$ George W. Kattawar, ${ }^{1}$ Ping Yang ${ }^{1,2}$, Michael S. Twardowski ${ }^{3}$, James M. Sullivan ${ }^{3}$

1. Department of Physics \& Astronomy, Texas A\&M University, College Station, TX 77843

2. Department of Atmospheric Sciences, Texas A\&M University, College Station, TX 77843

3. Harbor Branch Oceanographic Institute, Florida Atlantic University, Ft. Pierce, FL 34946

For publication in the Journal of Quantitative Spectroscopy \& Radiative Transfer

Corresponding author address: Dr. Bingqiang Sun, Department of Physics \& Astronomy, Texas A\&M University, College Station,TX 77843, USA. Email: bqsun@physics.tamu.edu 


\begin{abstract}
The optical properties of diatom chains in the ocean are studied based on a combination of the many-body iterative T-matrix (MBIT) method and an improved implementation of the ray-by-ray (RBR) geometric optics method. The MBIT, a numerically accurate method, is advantageous for scatterers with linear cells. In contrast to other popular geometric optics methods, the RBR, an approximate method, considers the interference of all outgoing rays. The two methods are verified in comparison with benchmark simulations. The simulation results of diatom chains in a wide size range can be obtained with either or both methods, and each can be applied to any optically soft particle, i.e., in the case when relative refractive index approaches unity.
\end{abstract}

Keywords: optical properties, diatom chains, many-body iterative T-matrix, ray-by-ray geometric optics method 


\section{Introduction}

The single-scattering properties of soft scatterers (i.e., the refractive index relative to the ambient medium approaches unity) have many practical applications in oceanic optical sensing, in particular, obtaining the characteristics of the scatterers, such as, the particle size distribution, shape, and refractive index by inversion from optical measurements [15]. The optical properties for all sizes of either homogeneous spherical scatterers or multi-layer concentric spherical shells can be analytically obtained in terms of the Lorenz-Mie theory [6-10]. Furthermore, the optical properties of an aggregate composed of multiple spheres can be analytically obtained through the multiple sphere T-matrix (MSTM) method [11]. For non-spherical scatterers with certain symmetries (e.g., completely or partially rotational), a semi-analytical method, the T-matrix, can be efficiently used to calculate the scattering properties, and two of the most effective realizations are the extended boundary condition method (EBCM) [12-14] and the invariant imbedding T-matrix (IITM) method $[15,16]$. The scattering properties of small sized scatterers can be obtained with numerically accurate methods, such as, the finitedifference time-domain (FDTD) $[17,18]$, the pseudo-spectral time-domain (PSTD) [19, 20], and the discrete dipole approximation [21-23]. For large sized scatterers, the geometric optics methods can be used to find the single-scattering properties [24-26].

In addition to the aforementioned scattering methods, some approximate methods, such as, Rayleigh-Gans scattering and the anomalous diffraction theory (ADT) [6], are appropriate for extremely soft scatterers, and numerous approximate methods have been employed to study soft scatterers [27-32]. One type of soft scatterer, the red blood cell, has been extensively studied using both accurate [33-37] and approximate methods [38- 
43]. The methods used for soft scatterers are only applicable to small-sized or extremely soft cases. In this study, the many-body iterative T-matrix (MBIT) $[44,45]$ and the geometric optics method using a volume integral equation of the electric field, the ray-byray (RBR) [24], are employed to obtain the scattering properties for a range of sizes for "soft" chain-forming triangular prism shaped diatoms found in the ocean.

\section{Methods}

\subsection{MBIT}

Based on the T-matrix, the incident and scattered electric field can be expanded in terms of the vector spherical wave functions [14]:

$$
\begin{aligned}
& \mathbf{E}^{i n c}(k \vec{r})=\sum_{n=1}^{\infty} \sum_{m=-n}^{n}\left[a_{m n} R g \mathbf{M}_{m n}(k \vec{r})+b_{m n} R g \mathbf{N}_{m n}(k \vec{r})\right] \\
& \mathbf{E}^{s c a}(k \vec{r})=\sum_{n=1}^{\infty} \sum_{m=-n}^{n}\left[p_{m n} \mathbf{M}_{m n}(k \vec{r})+q_{m n} \mathbf{N}_{m n}(k \vec{r})\right], r>r_{>}
\end{aligned}
$$

where $\mathrm{k}$ is the wave number in vacuum; $r$ is the radius of the smallest circumscribed sphere of a scatterer; $\operatorname{Rg} \mathbf{M}, \operatorname{RgN}, \mathbf{M}, \mathbf{N}$ are the vector spherical wave functions; and, $a_{m n}, b_{m n}$ and $p_{m n}, q_{m n}$ are the expansion coefficients of the incident and scattered fields [14]. Due to the linearity of the Maxwell equations, the transition matrix $\mathbf{T}$ can be formally expressed in terms of the relationship between the incident and scattered expansion coefficients:

$$
\left(\begin{array}{c}
p_{m n} \\
q_{m n}
\end{array}\right)=\sum_{n^{\prime}=1}^{\infty} \sum_{m^{\prime}=-n^{\prime}}^{n^{\prime}}\left(\begin{array}{cc}
T_{m n m^{\prime} n^{\prime}}^{11} & T_{m n n^{\prime} n^{\prime}}^{12} \\
T_{m n m^{\prime} n^{\prime}}^{21} & T_{m n m^{\prime} n^{\prime}}^{22}
\end{array}\right)\left(\begin{array}{l}
a_{m^{\prime} n^{\prime}} \\
b_{m^{\prime} n^{\prime}}
\end{array}\right)
$$

According to the MBIT method $[44,45]$, the original scatterer can be divided into $\mathrm{N}$ sub-scatterers along the direction of its main axis, and the T-matrix technique can deal 
with each sub-scatterer. Consequently, $\mathrm{N}$ frame of references exist, each located in the centers of the $\mathrm{N}$ sub-scatterers and enumerated from 1 to $\mathrm{N}$. The scattered field expansion coefficients of the original scatterer can be iteratively obtained in terms of the transition matrices of the sub-scatterers:

$$
\begin{aligned}
& \left(\begin{array}{c}
p_{m n}^{o(i)} \\
q_{m n}^{o(i)}
\end{array}\right)=\left(\begin{array}{c}
p_{m n}^{(i)} \\
q_{m n}^{(i)}
\end{array}\right)+\sum_{\substack{j=1 \\
j \neq i}}^{N} \sum_{n^{\prime}=1}^{\infty} \sum_{m^{\prime}=-n^{\prime}}^{n^{\prime}}\left(\begin{array}{cc}
R g A_{-m^{\prime} n^{\prime}}^{-m n}\left(k \vec{r}_{i j}\right) & \operatorname{Rg}_{-m^{\prime} n^{\prime}}^{-m n}\left(k \vec{r}_{i j}\right) \\
\operatorname{Rg} B_{-m^{\prime} n^{\prime}}^{-m n}\left(k \vec{r}_{i j}\right) & \operatorname{Rg}_{-m^{\prime} n^{\prime}}^{-m n}\left(k \vec{r}_{i j}\right)
\end{array}\right)\left(\begin{array}{c}
p_{m^{\prime} n^{\prime}}^{(j)} \\
q_{m^{\prime} n^{\prime}}^{(j)}
\end{array}\right), i=1, \ldots, N \\
& \left(\begin{array}{c}
p_{m n}^{(j)} \\
q_{m n}^{(j)}
\end{array}\right)=\sum_{n^{\prime}=1}^{\infty} \sum_{m^{\prime}=-n^{\prime}}^{n^{\prime}}\left(\begin{array}{cc}
T_{m n m^{\prime} n^{\prime}}^{11,(j)} & T_{m m m^{\prime} n^{\prime}}^{12,(j)} \\
T_{m n m^{\prime} n^{\prime}}^{21,(j)} & T_{m n m^{\prime} n^{\prime}}^{22,(j)}
\end{array}\right)\left(\begin{array}{c}
a_{m^{\prime} n^{\prime}}^{(j)} \\
b_{m^{\prime} n^{\prime}}^{(j)}
\end{array}\right), j=1, \ldots, N \\
& \left(\begin{array}{c}
a_{m n}^{(i)} \\
b_{m n}^{(i)}
\end{array}\right)=\left(\begin{array}{c}
a_{m n}^{o(i)} \\
b_{m n}^{o(i)}
\end{array}\right)+\sum_{\substack{j=1 \\
j \neq i}}^{N} \sum_{n^{\prime}=1}^{\infty} \sum_{m^{\prime}=-n^{\prime}}^{n^{\prime}}\left(\begin{array}{cc}
A_{-m^{\prime} n^{\prime}}^{-m n}\left(k \vec{r}_{i j}\right) & B_{-m^{\prime} n^{\prime}}^{-m n}\left(k \vec{r}_{i j}\right) \\
B_{-m^{\prime} n^{\prime}}^{-m n}\left(k \vec{r}_{i j}\right) & A_{-m^{\prime} n^{\prime}}^{-m n}\left(k \vec{r}_{i j}\right)
\end{array}\right)\left(\begin{array}{c}
p_{m^{\prime} n^{\prime}}^{(j)} \\
q_{m^{\prime} n^{\prime}}^{(j)}
\end{array}\right), i=1, \ldots, N
\end{aligned}
$$

where the superscript " $o$ " represents the expansion coefficients of the original scatterer; the superscripts " $i$ and $j$ " indicate the expansion coefficients obtained relative to the $\mathrm{i}$-th and $\mathrm{j}$-th frame of reference; $T^{(j)}$ is the $\mathrm{T}$-matrix of the $\mathrm{j}$-th sub-scatterer relative to its frame of reference; and, $\operatorname{RgA}, \operatorname{RgB}, \mathrm{A}$, and $\mathrm{B}$ are the addition coefficients, which represent the translation of the expansion coefficients between different frames of reference [46-48]. In Eqs. (2) and (4), the IITM is used to calculate the transition matrix of the sub-scatterers, and in Eq. (5), the stabilized bi-conjugate gradient iterative method is employed to accelerate the convergence rate [49-51]. The single-scattering properties of the original scatterer can be obtained in terms of Eqs. (3), (4), and (5).

\subsection{RBR}

When the characteristic length of a scatterer is much larger than the incident wavelength, the geometric optics method can be employed to find the internal field in terms of the ray 
tracing technique. Assuming each ray to have a circular cross section within the scatterer, the internal electric field $\mathbf{E}\left(\vec{r}^{\prime}\right)$ can be obtained in terms of the superposition of the electric field values associated with various rays within the particle, that is, the total internal electric field (the near field) can be added ray by ray [24]. The scattered electric field in the far field can be obtained in terms of the volume integral equation:

$$
\left.\mathbf{E}^{s c a}(k \vec{r})\right|_{k r \rightarrow \infty}=\frac{\exp (i k r)}{-i k r} \frac{-i k^{3}}{4 \pi} \iiint_{V}(\epsilon-1)\left[\mathbf{E}\left(\vec{r}^{\prime}\right)-\hat{r}\left(\hat{r} \cdot \mathbf{E}\left(\vec{r}^{\prime}\right)\right)\right] \exp \left(-i k \hat{r} \cdot \vec{r}^{\prime}\right) d^{3} r^{\prime}
$$

where $\mathbf{E}\left(\vec{r}^{\prime}\right)$ is the internal field within the scatterer, $k$ is the wavenumber in vacuum, $\hat{r}$ is the unit vector of the scattering direction, and $\epsilon$ is the isotropic permittivity of the scatterer. The volume integral equation in Eq. (6) can be replace by a summation over number of rays and scattering orders in a geometric optics approximation as follows:

$$
\left.\mathbf{E}^{s c a}(k \vec{r})\right|_{k r \rightarrow \infty}=\frac{\exp (i k r)}{-i k r} \frac{i k^{3}}{4 \pi} \sum_{\gamma} \sum_{p}(1-\epsilon) q_{\gamma, p}(\hat{r}) \mathbf{U}_{\gamma, p} \mathbf{E}^{i n t}
$$

where $\gamma$ denotes the number of rays, which can cover all the illuminated side of the scatterer, $\mathrm{p}$, from 0 to $\infty$, denotes the scattering order, where the incident point is defined as order 0 and then order from 1 to $\infty ; \mathbf{E}^{\text {int }}$ is the incident field; $\mathbf{U}_{\gamma, p}$ is the Fresnel coefficient associated with a series of reflection and refraction processes; $q_{\gamma, p}$ is the phase integral associated with the phase of electric field and the scattering direction over the volume past by a ray $\gamma$ from the order $p$ to the order $(p+1)$. The single scattering properties can be obtained by Eq. (7) in terms of a ray-by-ray algorithm (RBR). The RBR actually is a geometric-physical optics method, where the near field is obtained by raytracing and the far field by accurately mapping the near field to in terms of the Eqs. (6) and (7). Moreover, implicitly included into the calculation is the diffraction, a significant 
component of forward scattering for soft scatterers [40]. In Eq. (7), the interferences of the diffraction and the different rays are all automatically taken into consideration, which is important for the scattering of soft scatterers [43]. For homogeneous facet scatterers, the ray-tracing process can be obtained analytically by the physical-geometric optics hybrid (PGOH) method [26], which can be used to verify the RBR process.

\section{Simulation results and discussion}

Diatoms, a type of ocean phytoplankton, are a significant part of the oceanic ecosystem. In 3D in situ holographic imaging observations [52-54], some diatoms not only show large aspect ratios, but also link together to form linear chains. In a plethora of diatom cell shapes, the present simulations of the optical properties of chain-forming triangular prism diatoms are focused on the Mueller Matrix elements $[6,7]$.

Figure 1 shows images of the triangular prism Ditylum sp.: (a) of linear chains in the ocean where they tend to align in the direction of the ocean currents, (b) of a single chain, and (c) of a single diatom cell. A single cell is composed of three layers: the outer hard shell with two spines, the cytoplasm, and the cell nucleus. Based on the materials of the three layers, the refractive indices relative to water are respectively set to be $1.1+\mathrm{i} 0.0$, $1.03+\mathrm{i} 0.0001$, and $1.06+\mathrm{i} 0.025[55,56]$.

Figure 2 (a) shows the simulation model in terms of a diatom cell structure with dimensions taken from microscopic analysis with two concentric triangular prisms for the two outer layers, two cylinders for the two spines, and a sphere in the center for the core. Diatom chains are formed by linearly connecting the cells. Figure 2 (b) shows the laboratory frame of reference with the z-axis along the prism main axis and the $\mathrm{x}$-axis perpendicular to one of the prism side surfaces and pointing inside toward the 
corresponding edge of the prism, and the interpretation of the incident relative angles. $\mathrm{H}$ indicates the prism height and $\mathrm{r}$ is the distance from the prism lower center to the vertex.

\subsection{Validation of the present computational methods}

The transition matrix is necessary input for the MBIT and is indicated by Eq. (4). The IITM is employed to find the T-matrix of the inhomogeneous diatom cell shown in Figure 2. The Amsterdam discrete dipole approximation (ADDA) code is used as a benchmark method to validate the simulations.

Figure 3 shows a comparison between the Mueller matrix elements computed with the IITM and the ADDA for the diatom cell shown in Figure 2(a). In Figure 2(b), the outer prism height $\mathrm{H}$ and radius $\mathrm{r}$ are $3 \mu \mathrm{m}$ and $0.75 \mu \mathrm{m}$, the inner prism height $\mathrm{H}$ and radius $\mathrm{r}$ are $2.86 \mu \mathrm{m}$ and $0.68 \mu \mathrm{m}$, the core radius is $0.19 \mu \mathrm{m}$, and the spine length and diameter are $0.6 \mu \mathrm{m}$ and $0.15 \mu \mathrm{m}$, the incident wavelength is $0.475 \mu \mathrm{m}$, and the incident angles are $\theta_{I}=40^{\circ}, \phi_{I}=30^{\circ}$. The results computed from the two methods are identical, and indicate the MBIT can successfully use the transition matrix to obtain the singlescattering properties of many diatom cells.

Figure 4 shows a comparison between the Mueller matrix elements computed with the MBIT and the ADDA for two diatom cells. Two diatom cells are linearly connected along the direction of the z-axis in the laboratory frame of reference. For each cell, all the parameters are the same as in Figure 3. The MBIT uses an iterative technique to calculate the scattering properties of the two diatom cells while the ADDA generates dipoles of the two diatom cells and treats them as a whole. The perfect agreement indicates the accuracy of the MBIT. 
Figure 5 shows a comparison between the Mueller Matrix elements computed with the PGOH and the RBR for the homogeneous triangular prism shown in Figure 2(b). The prism height and radius are $9 \mu \mathrm{m}$ and $2.25 \mu \mathrm{m}$, the refractive index is $1.1+\mathrm{i} 0.0$, the incident wavelength is $0.475 \mu \mathrm{m}$, and the incident angles are $\theta_{I}=40^{\circ}, \phi_{I}=40^{\circ}$. The $\mathrm{PGOH}$ and the RBR are both geometric optics methods, but the ray-tracing part of the $\mathrm{PGOH}$ is analytically obtained while the RBR uses a number of rays to approximate the incident cross section. Exact results for the homogeneous prism indicate the correctness of the RBR process.

\subsection{Simulation of the optical properties of diatom particles}

The relative refractive indices of a diatom cell are close to unity, and the interactions between different diatom cells are weak. If no interaction between the cells is assumed, the scattered electromagnetic field of different cells relative to a standard has a phase difference $\exp \left(i k_{0}\left(\hat{n}_{i}-\hat{n}_{s}\right) \cdot \vec{r}_{j 0}\right)$, where $k_{0}, \hat{n}_{i}, \hat{n}_{s}, \vec{r}_{j 0}$ are, respectively, the wavenumber in a vacuum, the unit vectors of the incident and scattering directions, and the displacement vector of the $\mathrm{j}$-th cell relative to the standard.

Figure 6 compares the Mueller Matrix elements of three cells calculated by the MBIT and IITM, plus the phase differences with the assumption of no interaction between the cells. For one cell, the outer prism height $\mathrm{H}$ and radius $\mathrm{r}$ are $3 \mu \mathrm{m}$ and $0.75 \mu \mathrm{m}$, the inner prism height $\mathrm{H}$ and radius $\mathrm{r}$ are $2.86 \mu \mathrm{m}$ and $0.68 \mu \mathrm{m}$, the core radius is $0.19 \mu \mathrm{m}$, the spine length and diameter are $0.6 \mu \mathrm{m}$ and $0.15 \mu \mathrm{m}$, and the incident wavelength is $0.475 \mu \mathrm{m}$. An average of 273 orientations are taken in order to do a random orientation average. The 
discrepancy between the numerically accurate and the assumed samples is negligible, which indicates the weak interactions between diatom cells.

Figure 7 compares the Mueller Matrix elements of one cell computed by the IITM and RBR. The outer prism height $\mathrm{H}$ and radius $\mathrm{r}$ are $6 \mu \mathrm{m}$ and $1.5 \mu \mathrm{m}$, the inner prism height $\mathrm{H}$ and radius $\mathrm{r}$ are $5.72 \mu \mathrm{m}$ and $1.36 \mu \mathrm{m}$, the core radius is $0.38 \mu \mathrm{m}$, the spine length and diameter are $1.2 \mu \mathrm{m}$ and $0.3 \mu \mathrm{m}$, the incident wavelength is $0.475 \mu \mathrm{m}$, and the incident angles are $\theta_{I}=90^{\circ}, \phi_{I}=0^{\circ}$. For the backscattering of $\mathrm{P}_{11}$, a high specular peak occurs due to the flat surface of the outer prism. The elements $P_{12} / P_{11}, \mathrm{P}_{22} / P_{11}, P_{33} / P_{11}, P_{44} / P_{11}$ have the features of soft scatterers.

Figure 8 shows a comparison between the Mueller Matrix elements of one cell calculated by the ADDA and the RBR. The outer prism height $\mathrm{H}$ and radius $\mathrm{r}$ are $9 \mu \mathrm{m}$ and $4.5 \mu \mathrm{m}$, the inner prism height $\mathrm{H}$ and radius $\mathrm{r}$ are $8.58 \mu \mathrm{m}$ and $4.29 \mu \mathrm{m}$, the core radius is $0.57 \mu \mathrm{m}$, the spine length and diameter are $1.8 \mu \mathrm{m}$ and $0.45 \mu \mathrm{m}$, the incident wavelength is $0.475 \mu \mathrm{m}$, and the incident angles are $\theta_{I}=90^{\circ}, \phi_{I}=45^{\circ}$. The phase function peaks are due to the geometric optics effect. The right four panels show the features of soft scatterers. The simulation results become more accurate with an increase in cell size.

Figure 9 shows a comparison between the Mueller Matrix elements of one cell calculated by the ADDA and the RBR. The incident angles are $\theta_{I}=70^{\circ}, \phi_{I}=0^{\circ}$, and the other parameters are the same as in Figure 8. The elements agree very well except for $P_{43} / P_{11}$ which has discrepancies in the scattering angles around $90^{\circ}$.

Figure 10 shows a comparison between the Mueller Matrix elements of one cell computed with the IITM and the RBR. The outer prism height $H$ and radius $r$ are $9 \mu \mathrm{m}$ 
and $2.25 \mu \mathrm{m}$, the inner prism height $\mathrm{H}$ and radius $\mathrm{r}$ are $8.58 \mu \mathrm{m}$ and $2.04 \mu \mathrm{m}$, the core radius is $0.57 \mu \mathrm{m}$, and the spine length and diameter are $1.8 \mu \mathrm{m}$ and $0.45 \mu \mathrm{m}$, and the incident wavelength is $0.475 \mu \mathrm{m}$. The incident zenith angle $\theta_{I}$ is $90^{\circ}$ and the result is averaged by the incident azimuthal angles $\phi_{I}$ from $0^{0}$ to $60^{\circ}$. In this case, the elements $P_{12} / P_{11}, P_{33} / P_{11}$, and $P_{44} / P_{11}$ show perfect Rayleigh scattering features.

Figure 11 shows a comparison between the Mueller Matrix elements of two diatom cells computed with the MBIT and the RBR. All the parameters are the same as in Figure 10 except two cells are considered. The RBR ray tracing occurs on only one cell, but the ray tracing part of the other cells can be obtained in terms of the periodic boundary conditions. The elements are similar to Figure 10 because the interactions between two cells are weak, and the same conclusion is reached as that shown in Figure 6.

The refractive indices of all three layers of a single cell are soft so that the elements $\mathrm{P}_{12} / \mathrm{P}_{11}, \mathrm{P}_{22} / \mathrm{P}_{11}, \mathrm{P}_{33} / \mathrm{P}_{11}$ and $\mathrm{P}_{44} / \mathrm{P}_{11}$ have show similar Rayleigh scattering features in small and intermediate sizes. It indicates that these elements in the case of soft refractive indices and intermediate sizes are not sensitive to its shape and inhomogeneity.

\section{Conclusion}

In this study, the scattering properties of the diatom chains in the ocean are simulated in terms of two numerically accurate methods, the IITM and the MBIT, and an approximate method, the RBR. The application of the three methods to diatom chains is verified in comparison with the simulations based on the ADDA and the PGOH methods. The particle model and the refractive indices are based on the actual shape and composition of diatoms. In order to obtain reasonable results, the interference between soft particles' outgoing rays must be taken into consideration. The MBIT effectively deals with linear 
diatom cell chains with the transition matrix of one cell and the iterative technique. The RBR method calculates the near field by using the ray-tracing technique, and the far field by using a volume integral equation that maps the near field to the far field. For multiple diatom cells, the RBR traces one cell and the result is applied to all other cells in terms of the periodic boundary conditions. The simulation results of the diatom chains are shown for different aspect ratios, sizes, incident angles, and diatom numbers. Compared to the results calculated by the accurate methods, the overall agreement of the Mueller matrix elements has been obtained by the RBR. For soft particles, the MBIT and the RBR can be combined to obtain the scattering properties for a complete range of sizes.

\section{Acknowledgement}

The authors thank that Tamotsu Nagumo allows us to use his Ditylum brightwelli figure in Fig. 1(c). The authors thank Drs. Maxim Yurkin and A.G. Hoekstra for the use of their ADDA code. The authors acknowledge the Texas A\&M Supercomputing Facility for computing resources. George Kattawar acknowledges support by the ONR MURI program (N00014-09-1-1054) and the National Science Foundation Grant \# OCE-1130906. Ping Yang acknowledges the support by a NASA grant (NNX11AK37G). Bingqiang Sun was supported by ONR Coastal Geophysics. M. Twardowski and J. Sullivan gratefully acknowledge support from the National Ocean Partnership Program, ONR Coastal Geophysics, and the Harbor Branch Oceanographic Institute Foundation. The authors thank the anonymous reviewers for constructive comments. 


\section{References}

[1] Sullivan JM, Twardowski MS, Donaghay PL, Freeman SA. Use of optical scattering to discriminate particle types in coastal waters. Applied Optics. 2005;44:1667-80.

[2] Twardowski MS, Boss E, Macdonald JB, Pegau WS, Barnard AH, Zaneveld JRV. A model for estimating bulk refractive index from the optical backscattering ratio and the implications for understanding particle composition in case I and case II waters. Journal of Geophysical Research: Oceans (1978-2012). 2001;106:14129-42.

[3] Boss E, Twardowski MS, Herring S. Shape of the particulate beam attenuation spectrum and its inversion to obtain the shape of the particulate size distribution. Applied Optics. 2001;40:4885-93.

[4] Agrawal YC, Pottsmith HC. Instruments for particle size and settling velocity observations in sediment transport. Marine Geology. 2000;168:89-114

[5] Twardowski MS, Lewis MR, Barnard AH, Zaneveld JRV. In-water instrumentation and platforms for ocean color remote sensing applications. Remote Sensing of Coastal Aquatic Environments: Springer; 2005. p. 69-100.

[6] Hulst HC, Van De Hulst HC. Light scattering by small particles: Courier Corporation; 1957.

[7] Bohren CF, Huffman DR. Absorption and scattering of light by small particles: Wiley-Vch; 2008.

[8] Mueller JL. The influence of phytoplankton on ocean color spectra. 1973.

[9] Kitchen JC, Zaneveld JRV. A three - layered sphere model of the optical properties of phytoplankton. Limnology and oceanography. 1992;37:1680-90. 
[10] Bernard S, Probyn TA, Quirantes A. Simulating the optical properties of phytoplankton cells using a two-layered spherical geometry. Biogeosciences Discussions. 2009;6:1497.

[11] Mackowski D, Mishchenko M. A multiple sphere T-matrix Fortran code for use on parallel computer clusters. Journal of Quantitative Spectroscopy and Radiative Transfer. 2011;112:2182-92.

[12] Waterman P. Matrix formulation of electromagnetic scattering. Proceedings of the IEEE. 1965;53:805-12.

[13] Waterman P. Symmetry, unitarity, and geometry in electromagnetic scattering. Physical review D. 1971;3:825.

[14] Mishchenko MI, Travis LD, Lacis AA. Scattering, absorption, and emission of light by small particles: Cambridge university press; 2002.

[15] Johnson BR. Invariant imbedding $\mathrm{T}$ matrix approach to electromagnetic scattering. Applied optics. 1988;27:4861-73.

[16] Bi L, Yang P, Kattawar GW, Mishchenko MI. Efficient implementation of the invariant imbedding T-matrix method and the separation of variables method applied to large nonspherical inhomogeneous particles. Journal of Quantitative Spectroscopy and Radiative Transfer. 2012.

[17] Yee K. Numerical solution of initial boundary value problems involving Maxwell's equations in isotropic media. Antennas and Propagation, IEEE Transactions on. 1966;14:302-7.

[18] Yang P, Liou K. Finite-difference time domain method for light scattering by small ice crystals in three-dimensional space. JOSA A. 1996;13:2072-85. 
[19] Liu Q. The PSTD algorithm: A time - domain method requiring only two cells per wavelength. Microwave and Optical Technology Letters. 1998;15:158-65.

[20] Liu QH. The pseudospectral time-domain (PSTD) algorithm for acoustic waves in absorptive media. Ultrasonics, Ferroelectrics and Frequency Control, IEEE Transactions on. 1998;45:1044-55.

[21] Purcell EM, Pennypacker CR. Scattering and absorption of light by nonspherical dielectric grains. The Astrophysical Journal. 1973;186:705-14.

[22] Draine BT. The discrete-dipole approximation and its application to interstellar graphite grains. The Astrophysical Journal. 1988;333:848-72.

[23] Yurkin MA, Hoekstra AG. The discrete dipole approximation: an overview and recent developments. Journal of Quantitative Spectroscopy and Radiative Transfer. 2007;106:558-89.

[24] Yang P, Liou K. Light scattering by hexagonal ice crystals: solutions by a ray-byray integration algorithm. JOSA A. 1997;14:2278-89.

[25] Yang P, Liou KN. Geometric-optics-integral-equation method for light scattering by nonspherical ice crystals. Applied Optics. 1996;35:6568-84.

[26] Bi L, Yang P, Kattawar GW, Hu Y, Baum BA. Scattering and absorption of light by ice particles: Solution by a new physical-geometric optics hybrid method. Journal of Quantitative Spectroscopy and Radiative Transfer. 2011;112:1492-508.

[27] Sharma SK, Sommerford DJ. Light scattering by optically soft particles: theory and applications: Springer Science \& Business Media; 2006. 
[28] Shepelevich NV, Prostakova IV, Lopatin VN. Light-scattering by optically soft randomly oriented spheroids. Journal of Quantitative Spectroscopy and Radiative Transfer. 2001;70:375-81.

[29] Rysakov VM. Light scattering by "soft" particles of arbitrary shape and size. Journal of Quantitative Spectroscopy and Radiative Transfer. 2004;87:261-87.

[30] Yang P, Zhang Z, Baum BA, Huang H-L, Hu Y. A new look at anomalous diffraction theory (ADT): Algorithm in cumulative projected-area distribution domain and modified ADT. Journal of Quantitative Spectroscopy and Radiative Transfer. 2004;89:421-42.

[31] Xu M, Lax M, Alfano RR. Anomalous diffraction of light with geometrical path statistics of rays and a Gaussian ray approximation. Optics letters. 2003;28:179-81.

[32] Roy A, Sharma SK. On the validity of soft particle approximations for the light scattering by a homogeneous dielectric sphere. journal of modern optics. 1996;43:2225-37.

[33] Lu JQ, Yang P, Hu X-H. Simulations of light scattering from a biconcave red blood cell using the finite-difference time-domain method. Journal of Biomedical Optics. 2005;10:024022-02402210.

[34] Nilsson AMK, Alsholm P, Karlsson A, Andersson-Engels S. T-matrix computations of light scattering by red blood cells. Applied optics. 1998;37:2735-48. [35] He J, Karlsson A, Swartling J, Andersson-Engels S. Light scattering by multiple red blood cells. Josa a. 2004;21:1953-61. 
[36] Bi L, Yang P. Modeling of light scattering by biconcave and deformed red blood cells with the invariant imbedding T-matrix method. Journal of biomedical optics. 2013;18:055001-.

[37] Yurkin MA, Semyanov KA, Tarasov PA, Chernyshev AV, Hoekstra AG, Maltsev VP. Experimental and theoretical study of light scattering by individual mature red blood cells by use of scanning flow cytometry and a discrete dipole approximation. Applied optics. 2005;44:5249-56.

[38] Lugovtsov AE, Priezzhev AV, Nikitin SY. Light scattering by arbitrarily oriented optically soft spheroidal particles: calculation in geometric optics approximation. Journal of Quantitative Spectroscopy and Radiative Transfer. 2007;106:285-96.

[39] Borovoi AG, Naats EI, Oppel UG. Scattering of light by a red blood cell. Journal of biomedical optics. 1998;3:364-72.

[40] Streekstra GJ, Hoekstra AG, Nijhof E-J, Heethaar RM. Light scattering by red blood cells in ektacytometry: Fraunhofer versus anomalous diffraction. Applied Optics. 1993;32:2266-72.

[41] Hammer M, Schweitzer D, Michel B, Thamm E, Kolb A. Single scattering by red blood cells. Applied Optics. 1998;37:7410-8.

[42] Lim J, Ding H, Mir M, Zhu R, Tangella K, Popescu G. Born approximation model for light scattering by red blood cells. Biomedical optics express. 2011;2:2784-91. [43] Priezzhev AV, Nikitin SY, Lugovtsov AE. Ray-wave approximation for the calculation of laser light scattering by transparent dielectric particles, mimicking red blood cells or their aggregates. Journal of Quantitative Spectroscopy and Radiative Transfer. 2009;110:1535-44. 
[44] Yan W-Z, Du Y, Wu H, Liu D, Wu B-I. EM scattering from a long dielectric circular cylinder. Progress In Electromagnetics Research. 2008;85:39-67.

[45] Sun B, Yang P, Kattawar GW. Many-Body Iterative T-matrix Method for Large Aspect Ratio Particles. Journal of Quantitative Spectroscopy and Radiative Transfer. 2013.

[46] Friedman B, Russek J. Addition theorems for spherical waves. Quart Appl Math. $1954 ; 12: 13-23$

[47] Stein S. Addition theorems for spherical wave functions. Quart Appl Math. 1961;19.

[48] Cruzan OR. Translational addition theorems for spherical vector wave functions. Q Appl Math. 1962;20:33-40.

[49] Fokkema DR. Enhanced implementation of BiCGstab (l) for solving linear systems of equations: Citeseer; 1996.

[50] Chaumet PC, Rahmani A. Efficient iterative solution of the discrete dipole approximation for magnetodielectric scatterers. Optics letters. 2009;34:917-9.

[51] Tang J, Shen Y, Zheng Y, Qiu D. An efficient and flexible computational model for solving the mild slope equation. Coastal Engineering. 2004;51:143-54.

[52] Sullivan JM. A Submersible Holographic Camera for the Undisturbed Characterization of Optically Relevant Particles in Water (HOLOCAM). DTIC Document; 2011.

[53] Katz J, Donaghay PL, Zhang J, King S, Russell K. Submersible holocamera for detection of particle characteristics and motions in the ocean. Deep Sea Research Part I: Oceanographic Research Papers. 1999;46:1455-81. 
[54] Talapatra S, Hong J, McFarland M, Nayak A, Zhang C, Katz J, et al. Characterization of biophysical interactions in the water column using in situ digital holography. Mar Ecol Prog Ser. 2013;473:29-51.

[55] Aas E. The refractive index of phytoplankton, Rep. 46, 61 pp. Inst Geophys, Univ of Oslo, Oslo, Norway. 1981.

[56] Aas E. Refractive index of phytoplankton derived from its metabolite composition. Journal of Plankton Research. 1996;18:2223-49. 


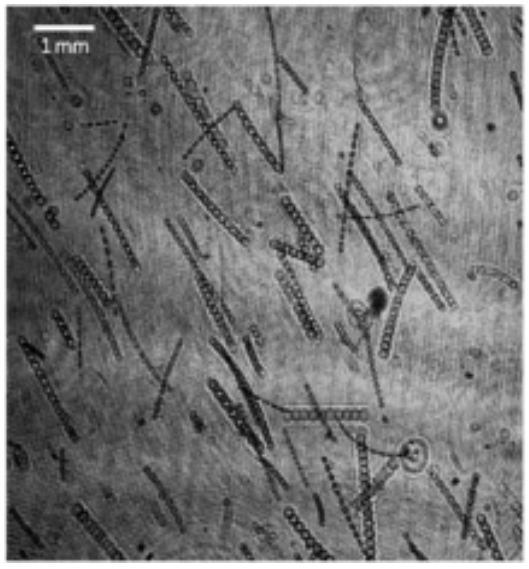

(a)

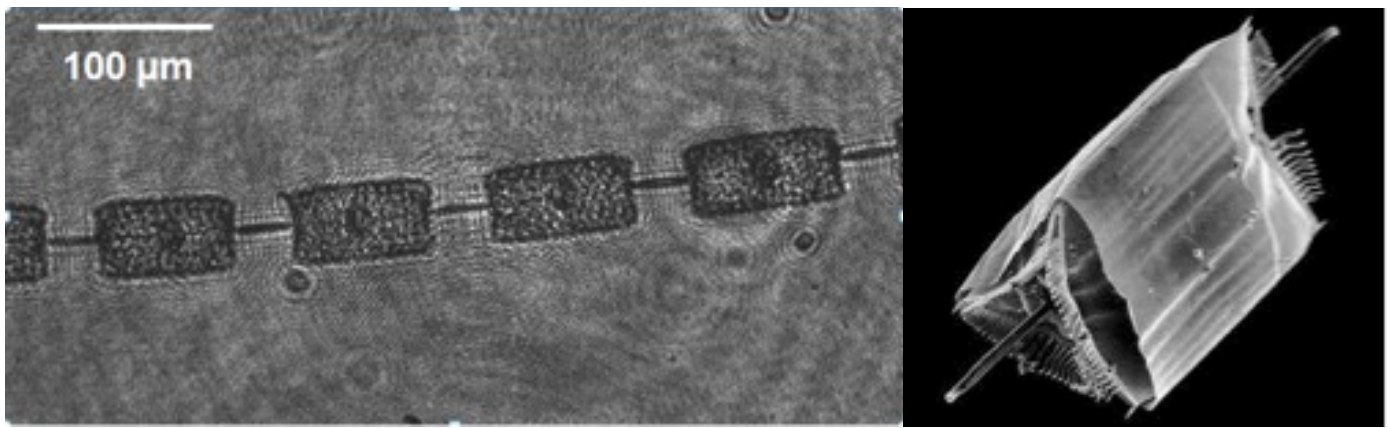

(b)

(c)

Figure 1. Different scale views of the diatom Ditylum: (a) linear chains of diatoms in the ocean; (b) a single diatom chain; and, (c) a single diatom cell. 


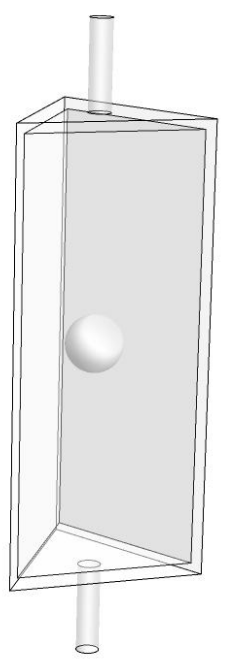

(a)

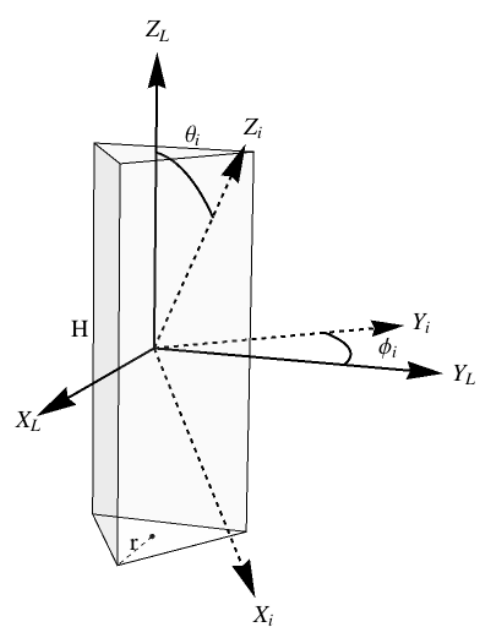

(b)

Figure 2(a). Simulation model in terms of diatom cell structure: two concentric triangular prisms represent the two outer layers and a center sphere represents the core. Chains are formed by linearly linking the diatom cells. (b). The laboratory frame of reference with the z-axis along the prism's main axis and the x-axis perpendicular to one of the prism side surfaces and pointing inside to the corresponding prism edge, and an interpretation of the relative incident angles. $\mathrm{H}$ indicates the prism height and $\mathrm{r}$ is the distance from the prism lower center to the vertex. 

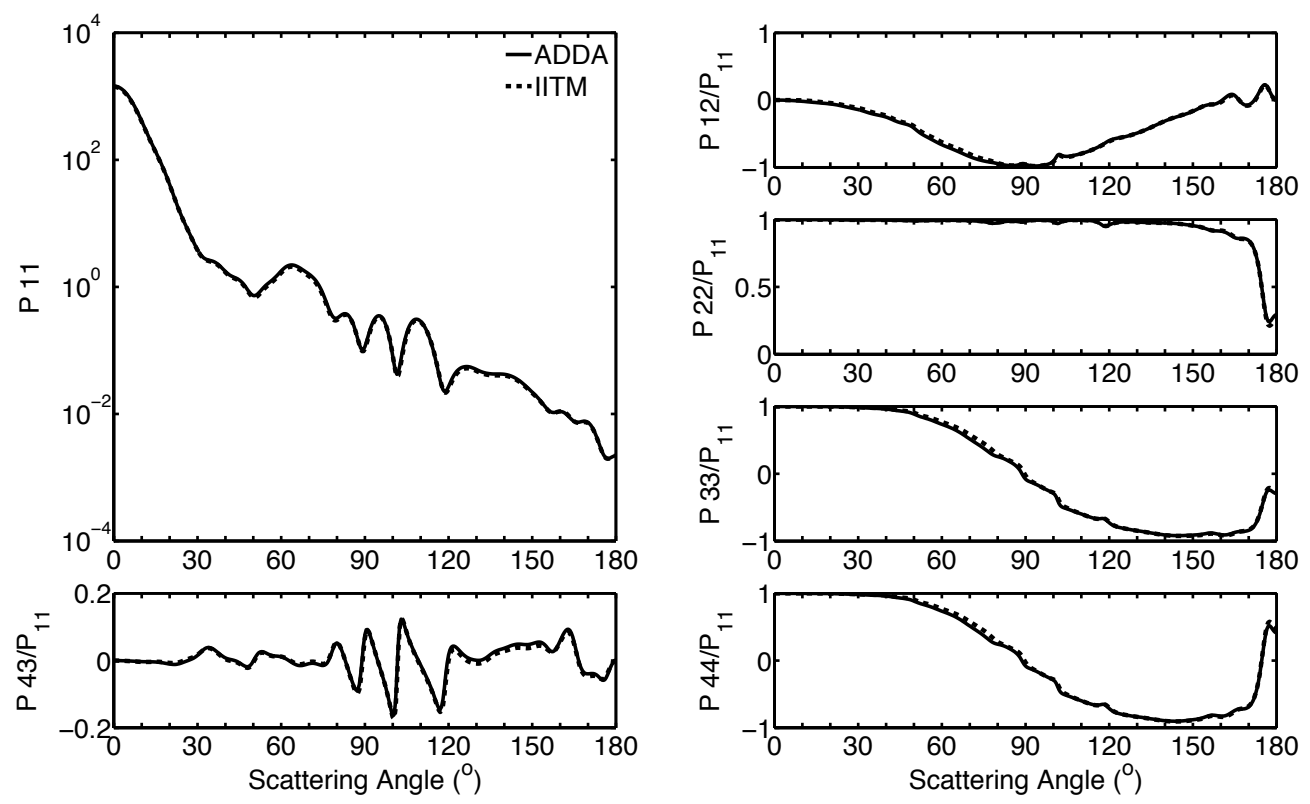

Figure 3. Comparison between the Mueller matrix elements calculated by the IITM and the ADDA for the diatom cell shown in Figure 2. The outer prism height $\mathrm{H}$ and radius $\mathrm{r}$ are $3 \mu \mathrm{m}$ and $0.75 \mu \mathrm{m}$, the inner prism height $\mathrm{H}$ and radius $\mathrm{r}$ are $2.86 \mu \mathrm{m}$ and $0.68 \mu \mathrm{m}$, the core radius is $0.19 \mu \mathrm{m}$, the spine length and diameter are $0.6 \mu \mathrm{m}$ and $0.15 \mu \mathrm{m}$, the incident wavelength is $0.475 \mu \mathrm{m}$, and the incident angles are $\theta_{I}=40^{\circ}, \phi_{I}=30^{\circ}$. 

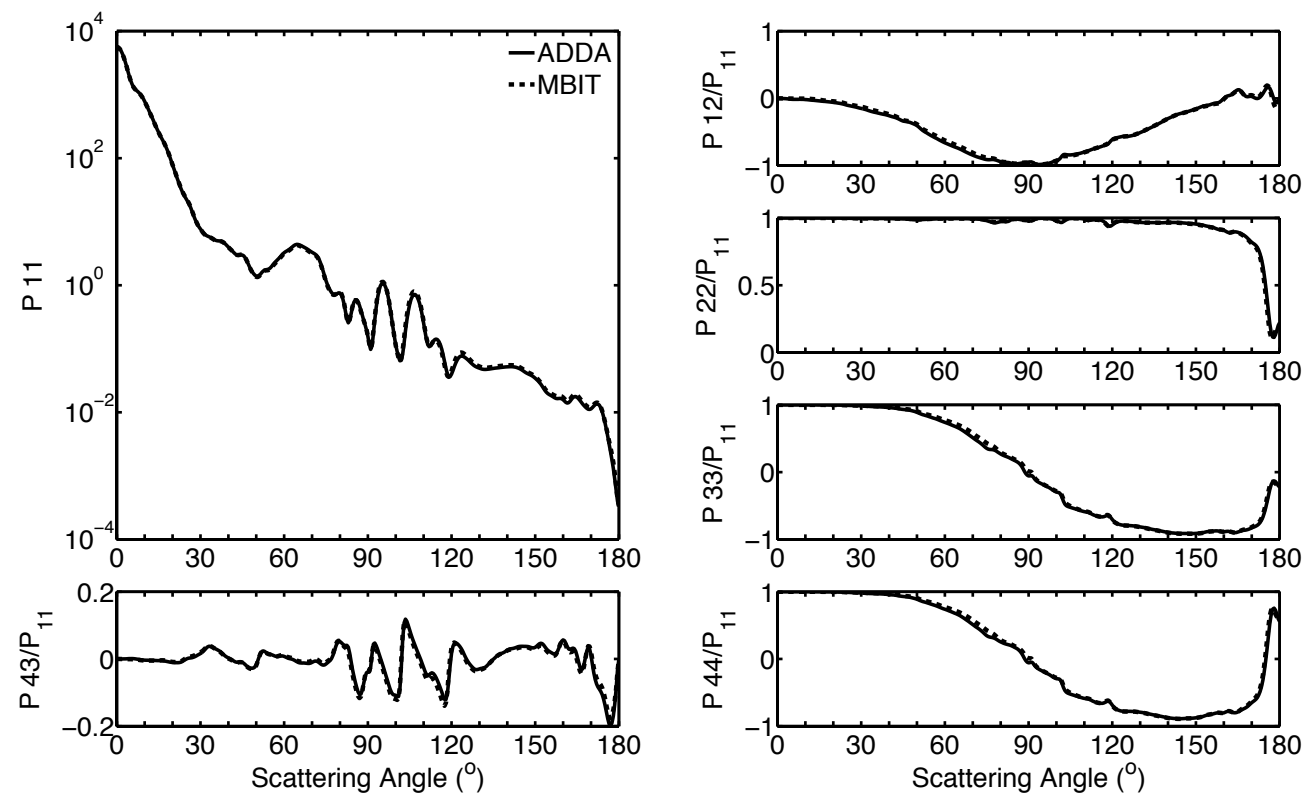

Figure 4. Comparison between the Mueller matrix elements of two diatom cells calculated by the MBIT and the ADDA. Two diatom cells are linearly connected along the direction of the z-axis of the laboratory frame of reference. For one cell, all the parameters are the same as in Figure 3. 

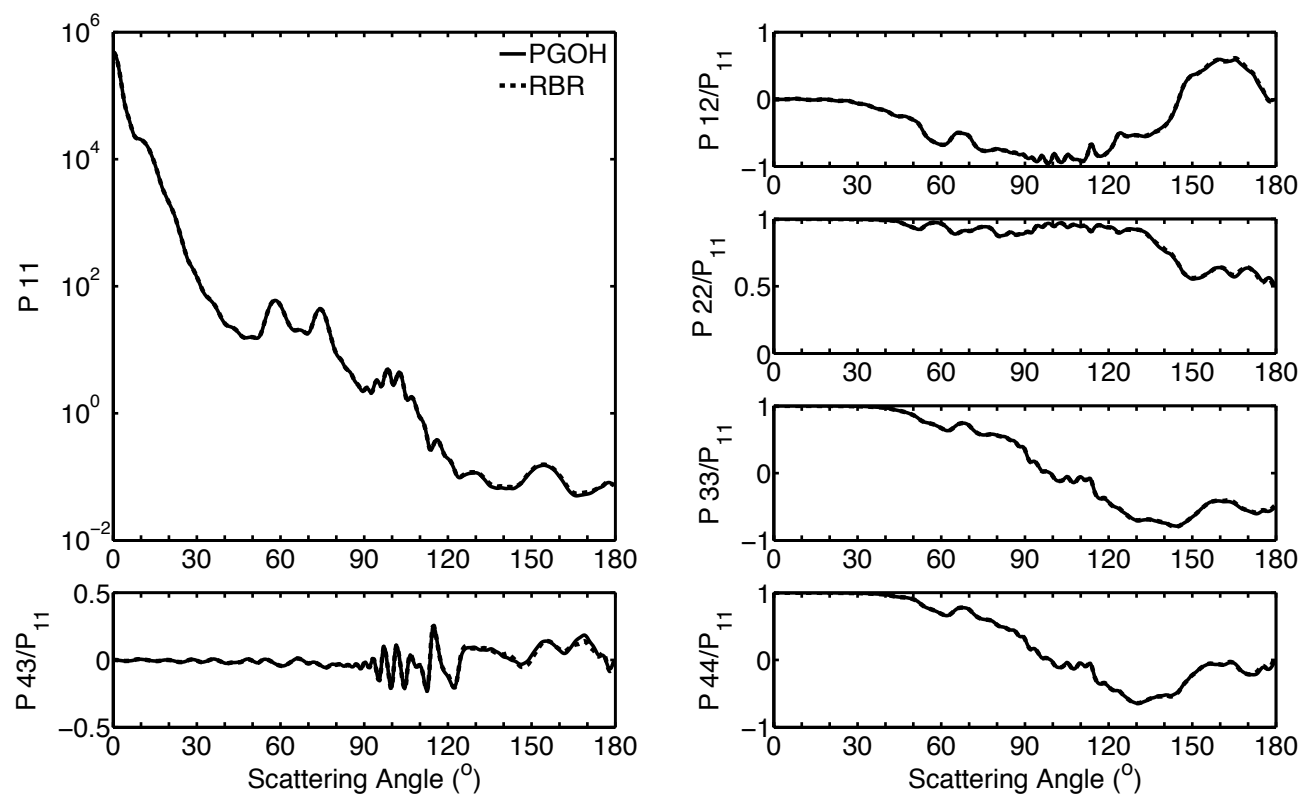

Figure 5. Comparison between the Mueller Matrix elements calculated by the PGOH and the RBR for the homogeneous triangular prism shown in Figure $2 \mathrm{~b}$. The prism height and radius are $9 \mu \mathrm{m}$ and $2.25 \mu \mathrm{m}$, the refractive index is $1.1+\mathrm{i} 0.0$, the incident wavelength is $0.475 \mu \mathrm{m}$, and the incident angles are $\theta_{I}=40^{\circ}, \phi_{I}=40^{\circ}$. 

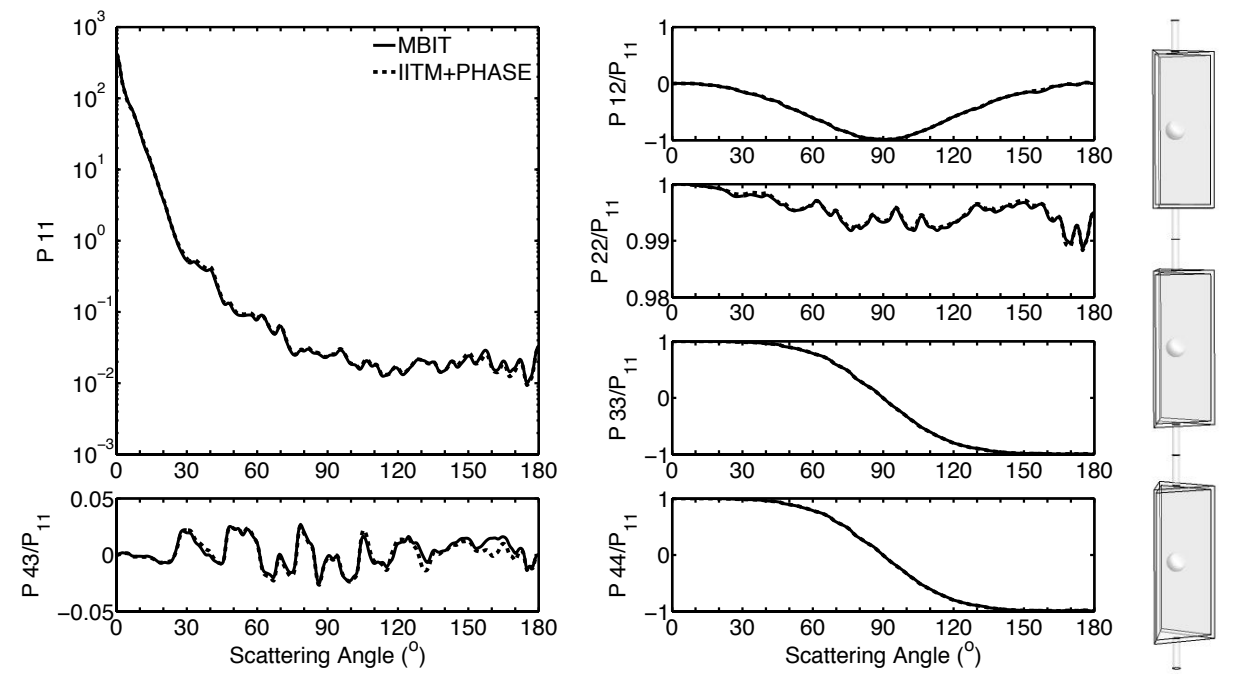

Figure 6. Comparison between the Mueller matrix elements of three cells calculated by the MBIT and by IITM, plus the phase difference with the assumption of no interaction between the cells. For one cell, the outer prism height $\mathrm{H}$ and radius $\mathrm{r}$ are $3 \mu \mathrm{m}$ and $0.75 \mu \mathrm{m}$, the inner prism height $\mathrm{H}$ and radius $\mathrm{r}$ are $2.86 \mu \mathrm{m}$ and $0.68 \mu \mathrm{m}$, the core radius is $0.19 \mu \mathrm{m}$, the spine length and the diameter are $0.6 \mu \mathrm{m}$ and $0.15 \mu \mathrm{m}$, and the incident wavelength is $0.475 \mu \mathrm{m}$. An average of 273 orientations are taken to obtain random orientation results. 

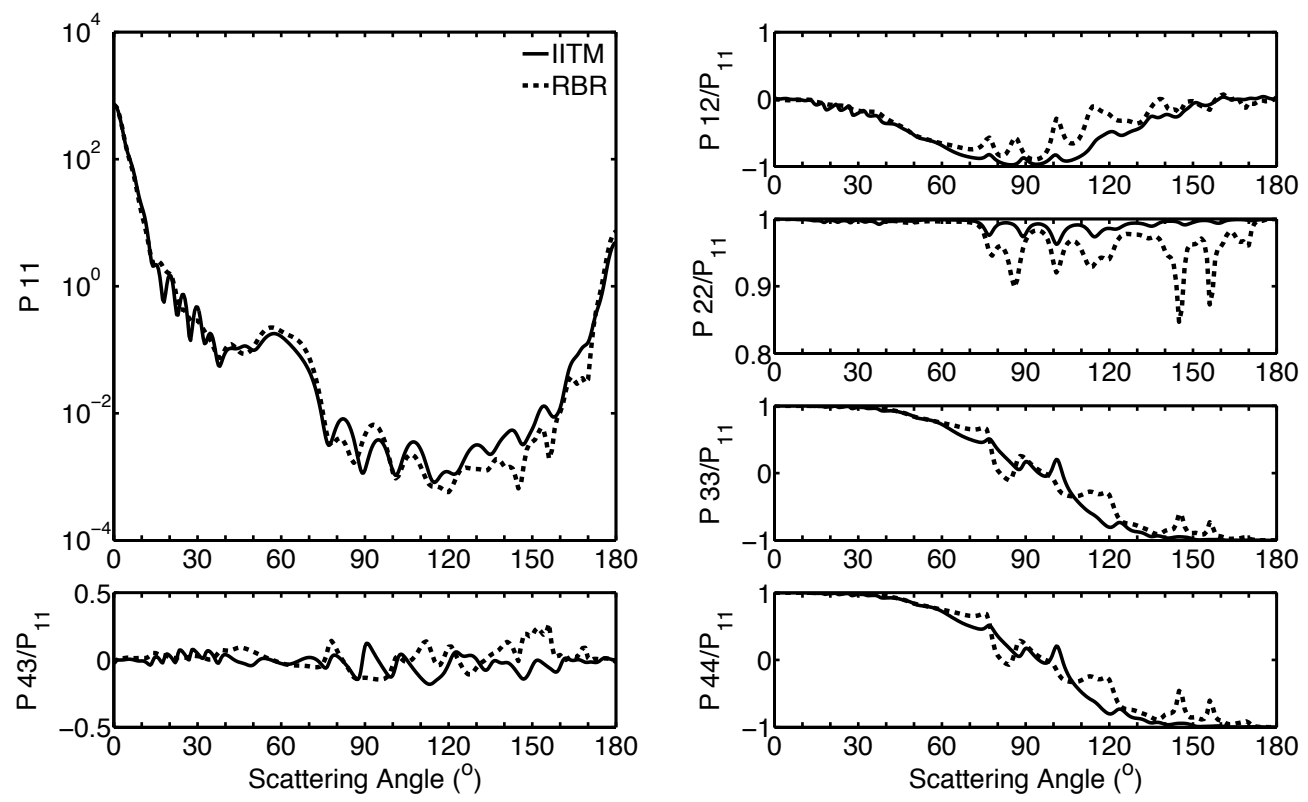

Figure 7. Comparison between the Mueller Matrix elements of one cell calculated by the IITM and RBR. The outer prism height $\mathrm{H}$ and radius $\mathrm{r}$ are $6 \mu \mathrm{m}$ and $1.5 \mu \mathrm{m}$, the inner prism height $\mathrm{H}$ and radius $\mathrm{r}$ are $5.72 \mu \mathrm{m}$ and $1.36 \mu \mathrm{m}$, the core radius is $0.38 \mu \mathrm{m}$, the spine length and diameter are $1.2 \mu \mathrm{m}$ and $0.3 \mu \mathrm{m}$, the incident wavelength is $0.475 \mu \mathrm{m}$, and the incident angles are $\theta_{I}=90^{\circ}, \phi_{I}=0^{\circ}$. 

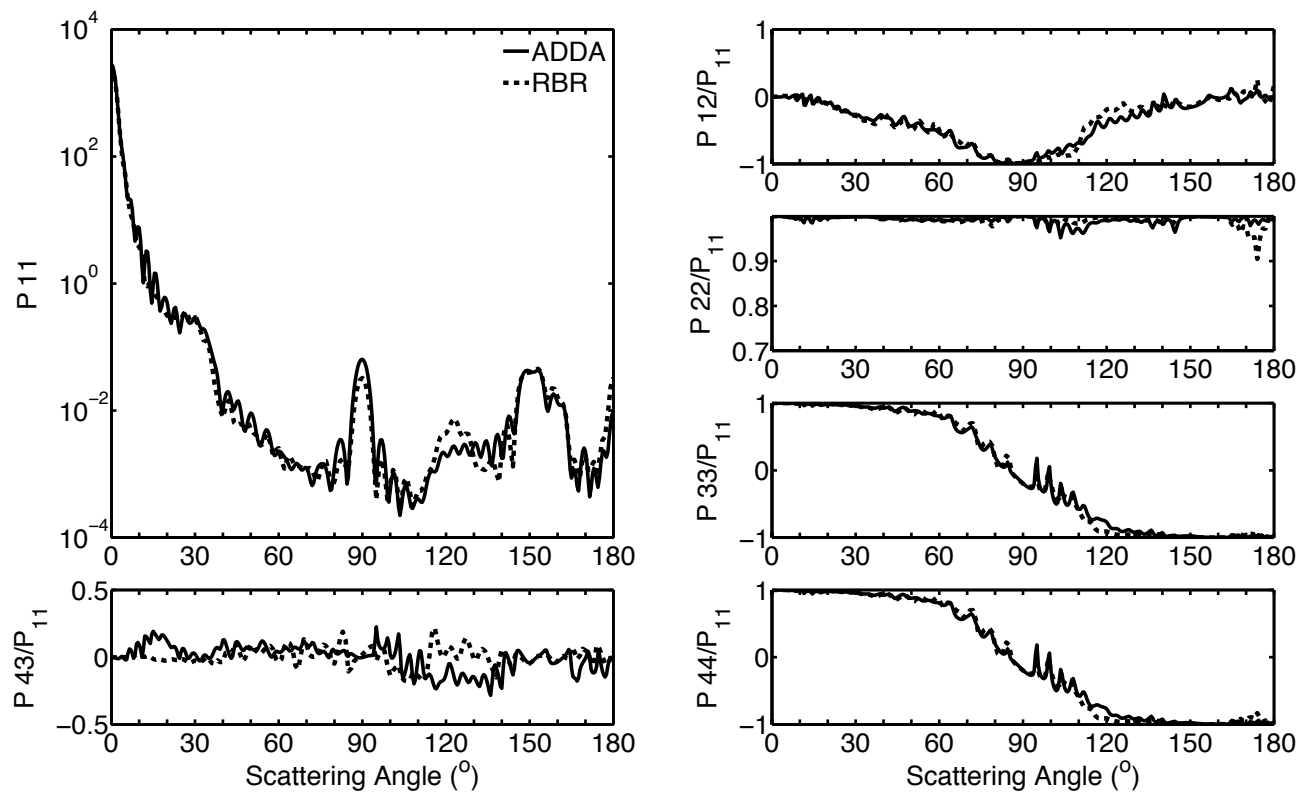

Figure 8. Comparison between the Mueller Matrix elements of one cell calculated by the ADDA and the RBR. The outer prism height $\mathrm{H}$ and radius $\mathrm{r}$ are $9 \mu \mathrm{m}$ and $4.5 \mu \mathrm{m}$, the inner prism height $\mathrm{H}$ and radius $\mathrm{r}$ are $8.58 \mu \mathrm{m}$ and $4.29 \mu \mathrm{m}$, the core radius is $0.57 \mu \mathrm{m}$, the spine length and diameter are $1.8 \mu \mathrm{m}$ and $0.45 \mu \mathrm{m}$, the incident wavelength is $0.475 \mu \mathrm{m}$, and the incident angles are $\theta_{I}=90^{\circ}, \phi_{I}=45^{\circ}$. 

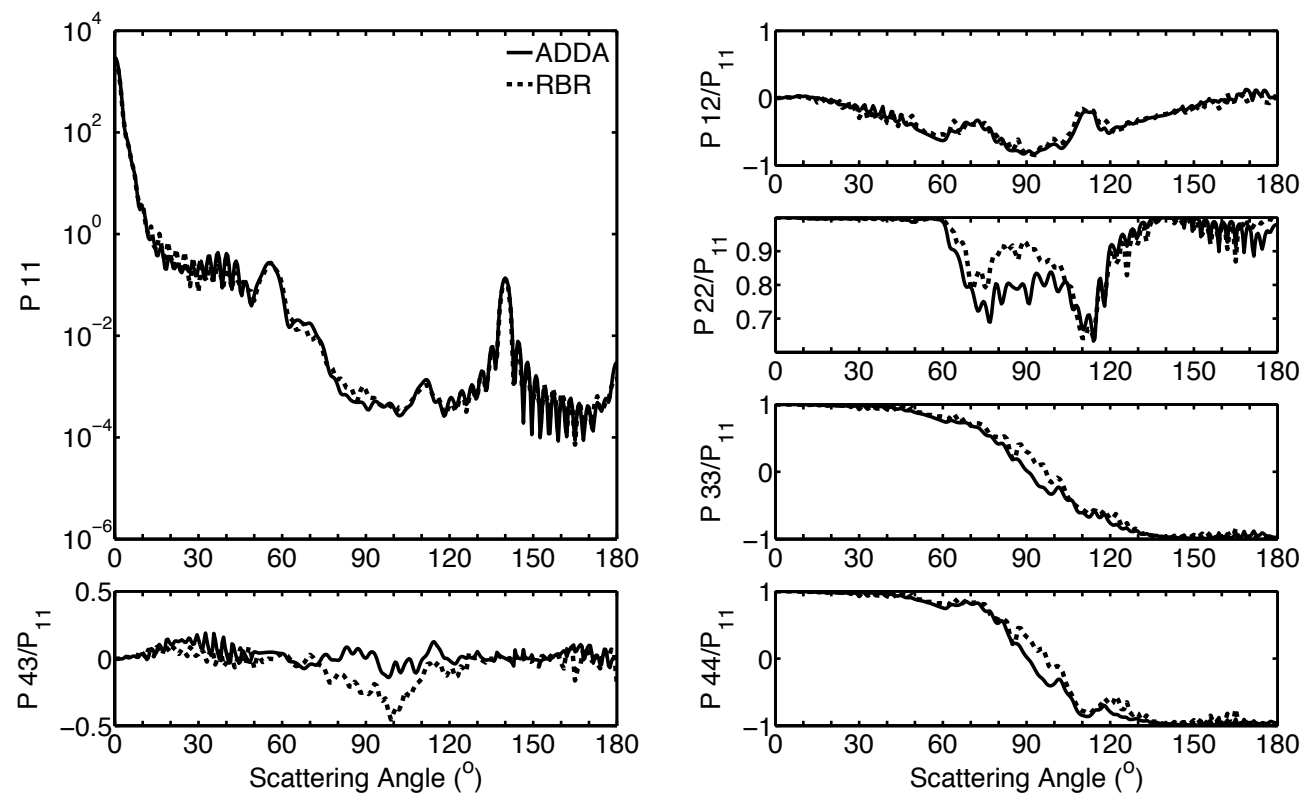

Figure 9. Comparison between the Mueller Matrix elements of one cell calculated by the ADDA and the RBR. The incident angles are $\theta_{I}=70^{\circ}, \phi_{I}=0^{\circ}$, and the other parameters are the same as in Figure 8. 

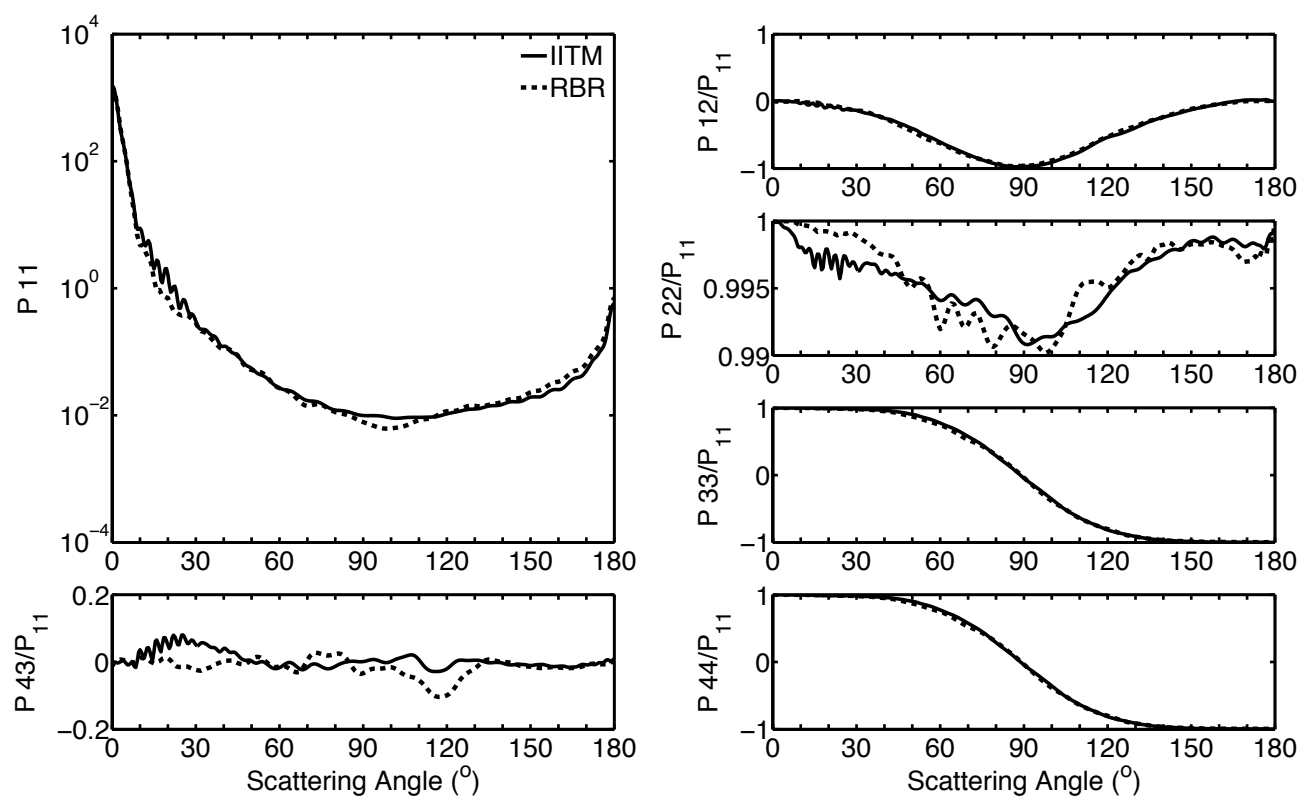

Figure 10. Comparison between the Mueller Matrix elements of one cell calculated by the IITM and the RBR. The outer prism height $\mathrm{H}$ and radius $\mathrm{r}$ are $9 \mu \mathrm{m}$ and $2.25 \mu \mathrm{m}$, the inner prism height $\mathrm{H}$ and radius $\mathrm{r}$ are $8.58 \mu \mathrm{m}$ and $2.04 \mu \mathrm{m}$, the core radius is $0.57 \mu \mathrm{m}$, and the spine length and diameter are $1.8 \mu \mathrm{m}$ and $0.45 \mu \mathrm{m}$, the incident wavelength is $0.475 \mu \mathrm{m}$, and the incident zenith angle $\theta_{I}$ is $90^{\circ}$ with the result averaged by the incident azimuthal angles $\phi_{I}$ from $0^{0}$ to $60^{\circ}$. 

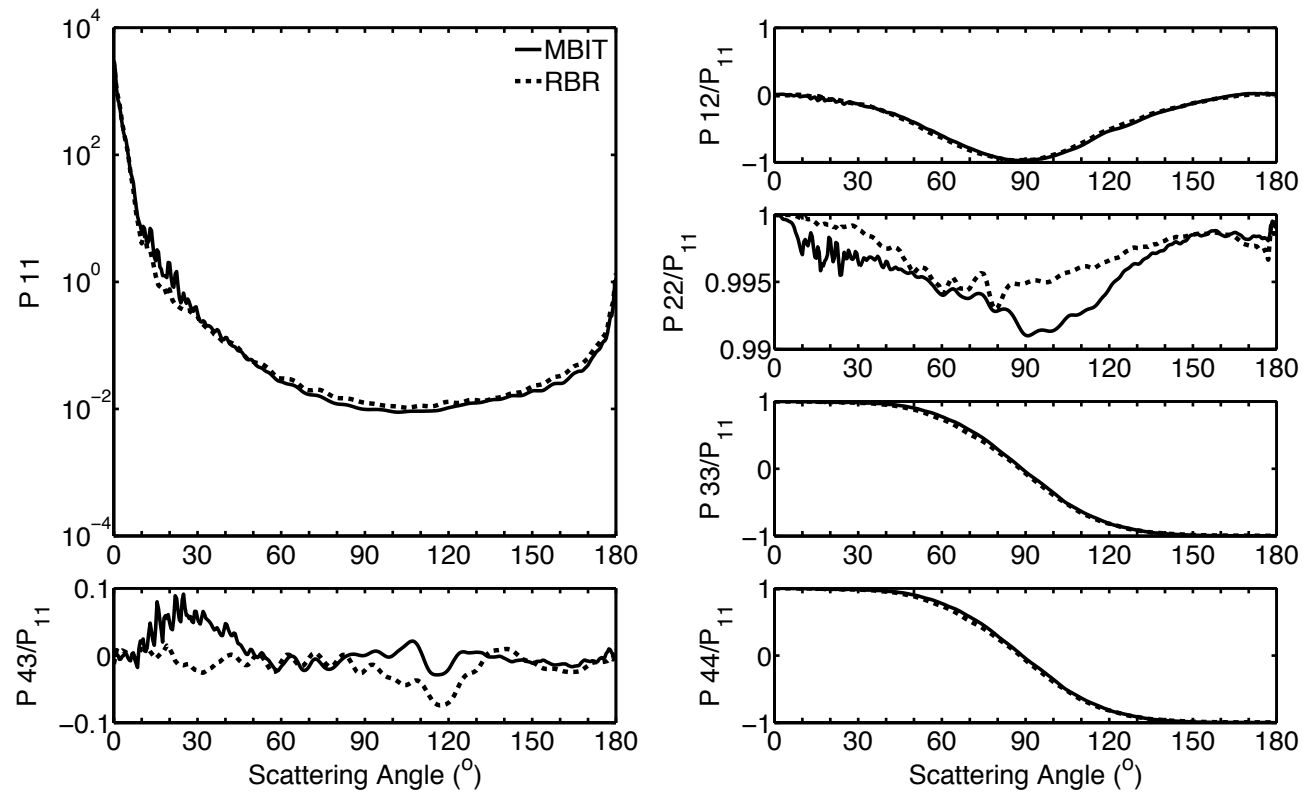

Figure 11. Comparison between the Mueller Matrix elements of two diatom cells calculated by the MBIT and the RBR. All the parameters are the same as in Figure 10 except that two cells are considered. 